\title{
Selective eye movements to simultaneously presented stimuli during discrimination'
}

\author{
STEPHEN R. SCHROEDER ${ }^{2}$ \\ UNIVERSITY OF NORTH CAROLINA
}

Human Ss, when given a discrimination task whose stimuli varied in dimension and relevance, always chose and fixated more frequently the stimulus they had been reinforced for choosing. Decreasing the brightness reduced the choice and fixation preference for form stimuli over line stimuli and raised total fixation frequency. Ss decreased fixation frequency to discriminanda with practice.

The macrosaccadic system is generally considered to be a servomechanism in a sampled data acquisition system (Robinson, 1968). The saccade is an efferently controlled instrumental response whose function is to centrate stimuli in the fovea so that information can be extracted more efficiently. When the eyes move from some stimulus fixated foveally to the next stimulus that was sampled in the periphery, the saccade and fixation perform a stimulus selection function by decreasing the detectability of stimuli at a distance from it.

This information-processing description of the saccadic system limits its analysis to the capabilities of the system (Yarbus, 1967), and the interaction of informational contents of peripheral and foveal stimuli. Most investigations thus far have involved such pattern recognition variables as stimulus novelty and complexity (Gould, 1967), affective tone (F aw \& Nunnally, 1967) or perceptual properties of the stimulus affecting its salience (Mackworth \& Morandi, 1967).

There is, however, another side to the macrosaccadic system. Since a saccade is an instrumental response, it is subject to reinforcement variables (Berger, 1968; Shroeder \& Holland, 1968a, b) and practice (Schaffer \& Gould, 1966). In such tasks as the discrimination of simple stimuli, one would expect variables like novelty and complexity to play less of a role in performance than reinforcement for looking at the critical components of a stimulus. Similarly, one might expect the sampling function of peripheral stimuli to be different during simple discrimination, since little new information is added to stimuli from trial to trial.

The present experiment was designed to discover whether or not fixations correspond to reinforced choices in a simple discrimination task and what effect practice would have on fixations. Nunnally, Stevens, and Hall (1965) have noted that first-grade children spent $22 \%$ more time in looking at geometrical forms that had previously been linked with rewards by experience with a slot machine device. Webb, Matheny, and Larson (1963) have demonstrated the usefulness of eye movements in the study of approach-avoidance conflict. Schaffer and Gould (1966) found that tachistoscopic practice resulted in faster scanning and fewer fixations per matrix in a digit-pattern recognition task. In the present experiment, it was expected that Ss would look most at those stimuli they were reinforced for choosing. Nevertheless, it was recognized that perceptual variables affect difficulty of discrimination. Therefore, the effect of brightness on the distribution of choices and fixations was also examined in an attempt to contrast the perceptual effects and reinforcement effects on differential fixation of the stimuli during discrimination.

\section{Subjects}

Eight female and seven male undergraduates from the University of Pittsburgh were assigned randomly to three groups. All had normal vision and were naive with respect to the task and apparatus.

\section{Apparatus}

The Ss were seated $28 \mathrm{in.} \mathrm{from} \mathrm{a}$ $7.5 \times 7.5$ in. Pola-Coat screen, on the corners of which the stimuli were projected from the rear. White stimuli $(1 \times 1$ in.) were projected into each comer ( $2 \times 2$ in.). Each stimulus subtended a visual angle of approximately $2 \times 2 \mathrm{deg}$ on a $4 \times 4 \mathrm{deg}$ background and was separated by $11 \mathrm{deg}$ (center to center) from the other stimuli. Bright stimuli were easily visible $(.90 \mathrm{~mL})$; dim stimuli were poorly discriminable (.45 mL).

Immediately behind the screen were six lights. In the middle were one green light to signify a correct choice and one red light to signify incorrect choice. One yellow light was next to each corner to indicate to the $S$ which choice he had made. The $S$ indicated his choice by pressing one of four buttons on a small response panel he held in his hand. One button corresponded to each corner of the screen and activated a yellow light next to its respective corner when depressed.

The eye-movement recording system was the Mackworth V-1 164-2 comeal reflection system with television digitizer (Polymetric Company) described by Schroeder and Holland (1968a). Frequency of eye movements, button presses, and correct response contingencies for each corner area were read out on an Esterline Angus Event Recorder.

\section{Procedure}

The $S$ was seated in front of the apparatus in a dental chair that was adjusted to make him comfortable. He then received the following instructions:

"This is a device for measuring pupil diameter. It works like this. A light shines off your left eye into this set of lenses and then into closed circuit TV where it is recorded and analyzed. To make the system work properly, it is necessary to hold your head as still as possible. If your head moves, errors are recorded. To help you hold your head still we use a bite plate with dental wax on it (make an impression for the S). When this hardens, I'll fasten it to the apparatus and that holds your head still. I can compensate for small movements by manipulating these cranks.

"Now I'11 explain what you can do. Figures will appear in the corners of this screen. Your job is to choose the correct figure. You indicate your choice by pressing one of these four buttons. Each button corresponds to one corner of the screen and, when pressed, turns on a yellow light next to the choice you made. If the choice you made is correct, a green light will appear in the center of the screen; if the choice is incorrect, a red light will appear. In the beginning you may have to guess a bit, but you shouldn't have any trouble figuring it out eventually."

The E then answered procedural questions; after that no further verbal feedback was given. A correct choice was followed immediately by a green light, a time-out lasting $3 \mathrm{sec}$, and an advance to the next slide. An incorrect choice was followed by a red light, a time-out of $30 \mathrm{sec}$ and no advance, i.e., the same slide kept reappearing until a correct choice was made. Each $S$ was paid $\$ 1.50$ at the end of the session.

The sequence of positions of each figure and the order of positive and negative 


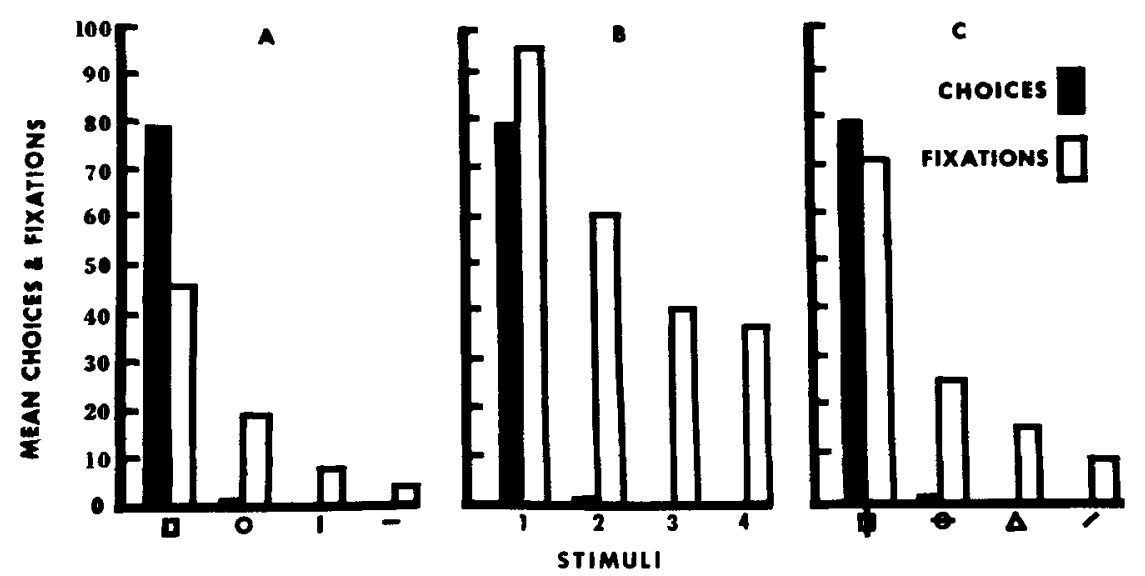

Fig. 1. Distributions of fixations and choices for Groups 1 (A), 2 (B), 3 (C) over sessions. Order of fixation frequency for all Group $1 \mathrm{Ss}$ was square, circle, vertical, horizontal line; for Group 2, positive chosen (square or vertical line), negative chosen stimulus (circle or horizontal line), nonchosen positive stimulus, nonchosen negative stimulus; for all Group 3 Ss, square with vertical line, circle with horizontal line, triangle, oblique line.

stimuli on each slide was counterbalanced both horizontally and vertically so that each figure appeared in each position in irregular sequence but an equal number of times for each block of 20 trials. This procedure was effective in precluding possible position preferences and requiring a scan when the next slide appeared.

Group 1 received one positive and one negative stimulus on each of two dimensions (form and line orientation). Square and vertical lines were positive; circle and horizontal lines were negative. These stimuli were bright.

Group 2 received the same treatment except that the stimuli were dim.

Group 3 received one positive stimulus (square with vertical line superposed), one negative stimulus (circle with horizontal line superposed), and two neutral stimuli (triangle and oblique line). The triangle and oblique line had no response consequences. Stimulus luminance was the same as for Group 1.

Thus Groups 1 and 2 could distribute their choices between two positive and two negative stimuli and ignore their dimensionality. Group 3 had only one positive and one negative stimulus to choose from, while two related but redundant stimuli have no response consequence.

\section{Distribution of Choices}

\section{RESULTS}

Distribution of choices to the stimuli is shown in Fig. 1. Fixation frequency here is the mean number of fixations of each shape per person for the entire session. Without exception, Ss chose one positive stimulus to the exclusion of all other stimuli regardless of whether there were one or two correct choices available. Once the $S$ was reinforced for choosing a stimulus, he continued to choose it on subsequent trials. All Ss in Groups 1 and 3 (Figs. $1 \mathrm{a}$ and $1 \mathrm{c}$ ) chose the square exclusively. In the dimmed stimulus condition (Group 2, Fig. 1b), three Ss chose the vertical and two $S s$ chose the square exclusively.

No $S$ made more than one error. Two Ss made one error to the circle in Group 1 . In Group 2, two Ss chose the horizontal line once and one $S$ chose the circle once. In Group 3, three Ss chose the circle with horizontal line once. In each case, the error occurred on the first trial and choice switched to the correct stimulus on the same dimension on the second trial. The other Ss performed the task without an error.

\section{Correspondence of Fixations to Choices} stimuli they were reinforced for choosing. Next, they fixated the stimulus they had chosen incorrectly, and they fixated least those stimuli they did not choose.

\section{Distribution of Fixations to Stimuli with Practice}

Figure 1 reveals another striking result: $S$ s did not fixate each stimulus on every trial. Ss without fail were looking at the feedback lights in the center of the display when the next slide appeared. In many cases, Ss chose the correct stimulus without scanning. Indeed, Ss in Group 1 chose the correct stimulus more than half of the time without removing their gaze from the center of the screen. Their general pattern was to scan fully on the first few trials; on subsequent trials they would fixate the square and circle before choosing, then only the square, and finally not at all.

Two repeated measures of analyses of variance (Lindquist, 1956) were performed on the fixation data to assess these patterns
In all cases (Fig. 1), Ss looked most at the of fixations. The first analysis companng Groups 1 and 2 had two within-groups variables (four stimuli and four 20-trial blocks) and one between-groups variable (bright vs dim). The differences between groups on the brightness variable failed to reach significance $(F=18.02, p<.10)$. The difference between stimuli was significant $(F=44, p<.005)$ as were the drop in fixation frequency over trials $(p<.05)$ and the Stimuli by Trial Block interaction $(\mathrm{p}<.05)$.

These results are clarified in Fig. 2, which shows mean fixation frequency of each stimulus over 20-trial blocks with all Ss included. Fixation of all stimuli decreased with practice, but fixations of chosen stimuli decreased at a faster rate since their initial level was much higher than that of nonchosen stimuli. Failure of the brightness variable to reach a significant difference was due to the small $\mathbf{N}$ and high individual difference in response patterns, which made the error terms in the analysis high. Figures $l a$ and $1 b$ show that the effect of dimming of the stimuli was actually to triple the fixation frequency while not altering the pattern of fixations.

A second analysis similar to the first, assessing the effects of number of reinforcing stimuli on fixation patterns was used for comparing Groups 1 and 3 . In this case, the difference between stimuli and drop in fixation frequency were again significant $(p<.01, p<.025$, respectively). Neither the difference between groups nor any of the interactions were significant.

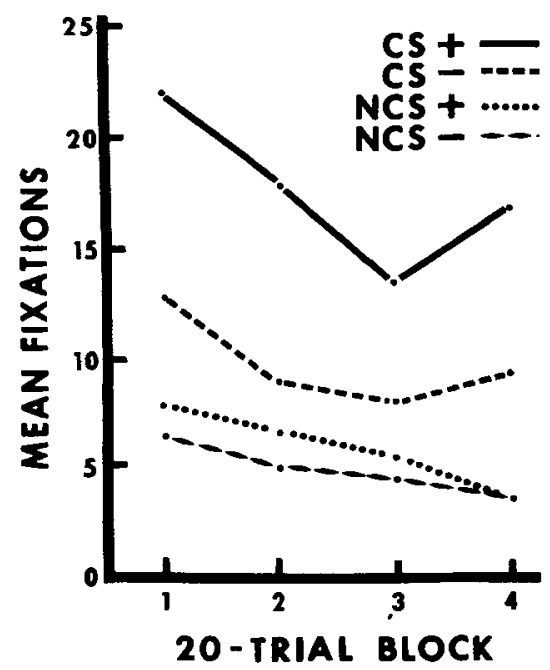

Fig 2. Distribution of fixations to the four stimuli (all three groups averaged) over 20-trial blocks. CSt is the positive chosen stimulus; $\mathrm{CS}-$, the negative chosen stimulus; NCS + , the nonchosen positive stimulus; NCS-, the nonchosen negative stimulus. 
Number of reinforcing stimuli available therefore did not affect fixation patterns.

\section{Distribution of Fixations to Chosen Stimulus Related to Quadrant Position}

A quadrant analysis was performed on the distribution of fixations to check for position preferences. Each quadrant was fixated about equally often. The mean number of fixations per person at each quadrant of the display was $34,34,30,33$. Data from individuals also show little difference.

The main results can be summarized: (1) When given a choice, Ss chose to respond to one reinforcing stimulus exclusively. (2) When given a choice between stimulus dimensions, Ss chose to respond to one stimulus dimension exclusively. (3) Decreased brightness reduced the preference for the form dimension over the line-orientation dimension. (4) Ss most of ten fixated those stimuli they had been reinforced for choosing. (5) In a simple discrimination situation, the effect of practice was to decrease fixations gradually to the discriminanda in an orderly pattern. (6) The effect of decreasing the brightness of the stimuli was to raise fixation frequency while not altering the pattern of fixation.

\section{DISCUSSION}

The results confirmed the expectations of the experiment. The most important variable affecting distribution of choices and fixations was reinforcement for choosing the correct stimulus. There were also indications that the dimensionality of the stimulus exerted some control over choices and fixations. Ss, when given a choice between reinforcing stimuli on different dimensions, fixated both relevant and redundant stimuli on one dimension more than the other.

It seems that the choice preference of form stimuli over line stimuli was due also to difference in their brightness. A reduction in brightness (Group 2) changed the choice preference from form to line, although form, shape, and position sequences for both Groups 1 and 2 were identical. In the bright stimulus condition, probably the difference in brightness of the form and line stimuli made the form stimuli more easily detectable through peripheral vision. The dim condition reduced this perceptual salience of form over line so that they were chosen about equally often. The fixation data support this interpretation. Figure 1b shows that Ss in the dim stimulus condition had to make more fixations to verify the location of the stimuli.

In this connection, it is interesting to note that, as trials progressed, fixations to different stimuli decreased in an orderly pattern for each S. Similarly, Schaffer and
Gould (1966) found for pattern recognition that increased practice resulted in a decreased fixation frequency of the stimuli. Premack and Collier (1966) and D'Amato, Etkin, and Fazzaro (1968) also found a decrease in observing during the course of discrimination learning. These two studies used the traditional observing response technique where $S$ presses a button that makes the discriminanda available.

This decrease is not unexpected. In all of the above experiments, including the present one, reinforcement was contingent upon cessation of observing. While knowledge of results was given, the discriminative stimuli were not available This coincidence discourages the making of redundant observing responses (D'Amato, Etkin, \& Fazzaro, 1968). Therefore, one would expect observing responses to decrease with practice to a point of maximum efficiency for choice performance.

D'Amato also found an augmentation of observing during reversal and extinction that was not easily accountable in terms of of conditioned reinforcement for looking. A related problem exists in the present experiment. Since all Ss were able to choose correctly without fixating the stimuli, why did they persist in fixating stimuli that were to them redundant after the first few trials? D'Amato's explanation, based on Berlyne's (1966) concept of exploratory motivation, seems also to be the most reasonable explanation in the present case. Motivation of exploratory perceptual behavior arises from lack of information. Lack of information leads to uncertainty and conflict, which results in exploratory responses that intensify stimulation from the environment. Thus $S s$ in the present experiment fixated all four stimuli on each trial early in the session. Soon they fixated mostly the stimuli on the dimension they were reinforced for choosing. Finally, on many trials they did not fixate the stimuli at all. The effect of practice, then, was to reduce uncertainty, until looking was minimally reinforcing.

This explanation, however, does not account for orderly pattern of stimulus selection. Each $S$ apparently imposed a gradient of fixation preference to the four stimuli (Fig. 1). This gradient was probably due to the perceptual properties of the stimuli that made them relevant to the reinforcing stimulus. This notion receives support from eye-movement studies in pattern recognition, where Gould (1967) found that Ss fixated more frequently dot patterns that were more similar to a matching standard. It thus appears that macrosaccades may be subject to stimulus generalization phenomena.

The above results also have a bearing on the function of saccades in visual information-processing models. It appears that a peripheral stimulus can initiate at least three types of eye-movement functions. (1) During familiarization, the peripheral stimulus may serve as a signal for a saccade (Robinson, 1968), so that subsequent foveal fixation can extract information more efficiently. (2) Suppression of irrelevant information, i.e., selection, may occur when information is gathered through peripheral vision and the foveally centrating saccade is either unnecessary or incompatible with efficient information gathering. (3) The peripheral stimulus may be a signal for refixation, and thus provide a verification or rehearsal function (Gould, 1967). All of these functions may change on a given task, depending on its perceptual, learning, memory, and reinforcement characteristics. The present experiment suggests, therefore, that, if any description of the macrosaccadic system is to have generality, it must also incorporate the effects of reinforcement and learning on eye movements.

\section{REFERENCES}

BERGER, R. J. Operant conditioning of eye movement in the monkey. Journal of the Experimental Analysis of Behavior, 1968, 11, $311-320$.

BERLYNE, D. E. Curiosity and exploration. Science, 1966, 153, 25-33.

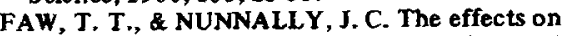
eye movement of complexity, novelty, and affective tone. Perception \& Psychophysics, 1967, 2, 263-267.

D'AMATO, M. R, ETXIN, M., \& FAZZARO, J. Cue-producing behavior in the Capuchin monkey during reversal, extinction, acquisition, and over-training. Journal of the Experimental Analysis of Behavior, 1968, 11, 425-434.

GOULD, J. D. Pattern recognition and eye-movement parameters. Perception \& Psychophysics, 1967, 2, 399-407.

LINDQUIST, E. F. Design and analysis of experiments in psychology and education. Boston: Hough ton Mifflin, 1956.

MACKWORTH, N. H. A stand ci nera for line-of-sight recording. Percep ion \& Psychophysics, 1967, 2, 119-227.

MACKWORTH, N. H., \& MORANDI, A. J. The gaze selects in formative details within pictures. Perception \& Psychophysics, 1967, 2, 547-551.

NUNNALLY, $J$., STEVENS, D., \& HALL, G. Association of neutral objects with rewards: Effect on verbal evaluation and eye movements. Journal of Experimental Child Psychology, $1965,2,4457$.

PREMACK, D., \& COLLIER, G. Duration of looking and number of looks as dependent variables. Psychonomic Science, 1966, 4, 81-82.

ROBINSON, D. A. Eye movement control in primates. Science, 1968, 161, 1219-1224.

SCHAFFER, A., \& GOULD, J. D. Eye movement patterns as a function of previous tachistoscopic practice. Perceptual \& Motor Skills, 1964, 19, 701-702.

SCHROEDER, S. R., \& HOLLAND, J. G. Operant control of eye movements. Journal of Applied Behavioral Analysis, 1968a, 1, 161-166.

SCHROEDER, S. R., \& HOLLAND, J. G. Operant control of eye movements during human vigilance. Science, 19685, 161, 292-293. 
TEICHNER, W. H. Interaction of behavioral and physiological stress reactions. Psychological Review, 1968, 75, 271-291.

TEICHNER, W. H., \& PRICE, L. M. Eye aiming behavior during the solution of visual patterns. Journal of Psychology, 1966, 62, 33-38.

WEBB, W., MATHENY, A., \& LARSON, G. Eye movements as a paradigm of approach and avoidance behavior. Perceptual \& Motor Skills, 1963, 16, 341-347.

YARBUS, A. L. Eye movements and vision. New York: Plenum, 1967.

\section{NOTES}

1. This research was supported by 0 .E. Contract No. 4-10-58.
2. Address: Department of Psychology, University of North Carolina, Chapel Hill, North Carolina 25714.

arolina

(Accepted for publication May 5, 1969.) 